\title{
Ridurre il dono alla donazione: II metodo fenomenologico e la teologia secondo Jean-Luc Marion
}

\author{
Reducing the gift to givenness: Phenomenological \\ method and theology according to Jean-Luc Marion
}

\section{Nicola Reali*}

Pontifícia Universidade Lateranense, Roma, Itália

\section{Riassunto}

La fenomenologia della donazione di Marion può rappresentare un'occasione di approfondimento del rapporto filosofia/teologia che cerchi di ricucire la frattura ormai secolare tra le due discipline? II presente articolo cerca di illustrare il progetto del filosofo francese - e l'obiettiva implicazione teologica del suo discorso - cercando di dettagliare una delle sue tesi centrali: la riduzione del dono alla donazione. Quest'ambito offre così lo spazio per una ricostruzione del percorso teoretico di Marion, che va di pari passo all'approfondimento del tema della paternità. Quest'ultima rappresenta l'exemplum privilegiato di che cosa significhi un dono ridotto alla donazione, poiché in essa Marion

\footnotetext{
NR: Doutor em Teologia, e-mail: reali@pul.it
} 
ritrova tutte le caratteristiche di ciò che la riflessione metodologica ha indicato in un dono ridotto alla donazione. La disamina del percorso disegnato da Marion favorisce pertanto l'occasione per evidenziare la necessità (teologica e filosofica) di un approfondimento del rapporto esperienza/riflessione che attende ancora una risposta soddisfacente.

Parole-chiave: Fenomenologia della donazione. Jean Luc Marion. Teologia e filosofia.

\begin{abstract}
Can Marion's phenomenology of givenness be an opportunity to get a deeper understanding of the relationship between philosophy and theology, an understanding that could mend the century-old rift between the two disciplines? This article aims to illustrate the project of the French philosopher - and the objective theological implication of his thought - by analyzing one of his central thesis: the reduction of the gift to givenness. This analysis allows the reconstruction of Marion's theoretical path, which goes along with the theme of fatherhood. The latter represents the privileged exemplum of what a gift reduced to givenness means, since Marion finds in it all the characteristics that the methodological thinking has indicated in a gift reduced to givenness. The examination of the course set by Marion raises, therefore, the opportunity to highlight the (theological and philosophical) need of deepening the understanding of the relationship between experience and reflection that still awaits a satisfactory answer.
\end{abstract}

Keywords: Phenomenology of givenness. Jean-Luc Marion. Theology and philosophy.

\title{
Introduzione
}

Presentare l'attenzione che la fenomenologia della donazione di Marion ha nei confronti della teologia, e parimenti tentare di interrogarsi sui possibili risvolti teologici del suo discorso, potrebbe sembrare un'ingenuità imperdonabile. 
Sicuramente lo sarebbe se l'approccio dimenticasse che Marion non persegue affatto un ricerca filosofica capace di interloquire con la teologia per un interesse, per così dire, "confessionale"; come accade, per esempio, quando si pretende che un cristiano filosofo sia per forza anche un filosofo cristiano. Altresì la semplice constatazione che entrambe le discipline spesso mettono a tema le stesse problematiche (la storia, il tempo, la libertà, etc), se fosse assunta come criterio del confronto filosofia/teologia tracciato da Marion, finirebbe anch'essa per restare irretita nelle maglie di una superficiale ingenuità. In Marion la questione è più radicale: la filosofia (meglio, la fenomenologia) si snaturerebbe se pregiudizialmente eliminasse dal suo campo di indagine i fenomeni rivelati (non solo quelli genericamente religiosi), e, nello stesso tempo, la teologia non sarebbe se stessa se non facesse proprio il confronto con la fenomenologia, anzi se non lo assumesse come proprio metodo. In questo caso, allora, l'ingenuità varrebbe a rilevare meglio quello che è un aspetto fondamentale dell'argomentare di Marion: l'essere convinto di superare lo sterile, e in definitiva malinconico, tentativo di rileggere le tematiche teologiche alla luce di questa o quella filosofia, per puntare, viceversa, ad individuare nel metodo fenomenologico stesso lo spazio entro il quale i fenomeni rivelati appaiono di diritto appartenenti al campo della fenomenalità così come ogni altro fenomeno (anzi, ne hanno maggior diritto proprio perché rivelati).

In gioco c'è pertanto la tensione a non accontentarsi di parlare filosoficamente di Dio, di Gesù Cristo o dell'esperienza cristiana, ma di mostrare come metodologicamente sia quasi necessario parlare di Dio, di Gesù Cristo e dell'esperienza cristiana se non si vuole relegare la fenomenologia ad un metodo che, come molti altri nel corso della modernità (e della post-modernità), hanno stabilito a priori dei limiti al campo della fenomenalità. Questa sfida è talmente accentuata in Marion che ogni parola dedicata a Dio, a Gesù Cristo e all'esperienza cristiana sarebbe letteralmente una parola inutile, se non servisse a mettere in evidenza la qualità della posta in gioco per il metodo fenomenologico, che è, appunto, quella di rovesciare la tradizionale subordinazione della fenomenalità ad una com-prensione a priori della stessa, per lasciare che i fenomeni manifestino - nella loro apparizione - nient'altro che essi stessi. In altri termini che l'apparizione dei fenomeni sia una rivelazione, ma una 
rivelazione che non mostra qualcosa d'altro, che non ci apre ad una esteriorità: una rivelazione che si dischiude a se stessa ${ }^{1}$.

La sfida, come si vede, è di quelle che mettono in gioco i termini fondamentali del secolare rapporto filosofia/teologia e che, proprio per la radicalità con cui pretende di riprecisare dall'interno il metodo fenomenologico, non può essere lasciata nel generico ambito delle dichiarazioni d'intenti, ma chiede una rigorizzazione teoretica che il filosofo francese non ha esitato a proporre (cf. MARION, 1989; 1997). In essa — partendo da un puntuale confronto con i padri fondatori del metodo fenomenologico (Husserl e Heidegger) - Marion ha indicato come prioritario il superamento di ogni visione pre-determinata del campo della fenomenalità in chiave sia antropologica sia ontologica. In pratica si tratta di considerare che per Marion ogni qual volta la datità dei fenomeni è subordinata ad un a priori di tipo antropologico od ontologico si osserva una pre-determinazione del campo della fenomenalità che porta con sé inesorabilmente una ricaduta nel formalismo trascendentale. Occorre impedire qualsiasi ri(con)duzione dell'apparizione dei fenomeni ad una condizione di possibilità antecedente alla loro donazione, poiché così facendo si produce una comprensione dei fenomeni stessi a partire da qualcos'altro rispetto alla loro donazione: «le "cose sensibili" appaiono e meritano il titolo di "fenomeni", ma esse lo devono ad una ragione altra, differente dalla loro apparizione (apparition) stessa, che sola è sufficiente a qualificare questa apparenza (apparence) come un fenomeno» (MARION, 1992, p. 82). Viceversa, per Marion, il darsi concreto dei fenomeni deve essere riconosciuto come l'impadroneggiabile antropologicamente (sia concettualmente, sia trascendentalmente) che nessun orizzonte di manifestazione può pre-determinare. Il fenomeno per Marion — riprendendo Sein und Zeit - è

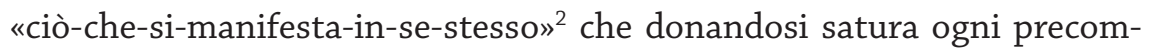
prensione antropologica e ogni orizzonte di manifestazione. Solo la donazione dei fenomeni rappresenta infatti l'unico criterio capace di consolidare l'itinerario della fenomenologia al di fuori delle strettoie poste a priori

\footnotetext{
1 Per una dettagliata analisi del rapporto filosofia/teologia nel pensiero di Marion rimandiamo al nostro Reali, N. Fino all'abbandono. L'eucaristia nella fenomenologia di Jean-Luc Marion. Roma: Città Nuova, 2001.

2 "das Sich-an-ihm-selbst-zeigende": HEIDEGGER, M. Sein und Zeit. In HGA, Bd. II, Frankfurt a. M.: Klostermann, 1977, p. 31.
} 
dal pensiero moderno al campo della fenomenalità, poiché la presa in considerazione della datità (Gegebenheit) dei fenomeni — non separatamente dal gesto fenomenologico per eccellenza della riduzione - permette di scorgere in ciò che appare l'apparire in quanto tale (l'apparaissant comme tel), così che la datità stessa non si riduce alla sua apparenza (apparence), ma alla sua donazione (Gegebenheit): «tanta riduzione, quanta donazione (autant de réduction, autant de donation)» (MARION,1997, p. 23) ${ }^{3}$. Questa donazione al di là dell'essere e del soggetto è anche ciò che determina una visione impregiudicata della "soggettività" stessa poiché il carattere donato dei fenomeni dice l'impossibilità di specificare l'identità del "soggetto" a monte dell'atto della donazione dei fenomeni, i quali dandosi - donano sia se stessi sia il loro riconoscimento antropologico. In quest'ultimo il "soggetto" non solo riconosce il fenomeno dato come donato, ma si ri-conosce: egli è colui che è costretto a rinunciare ad ogni attività costituente nei confronti della manifestazione del dato, il quale, in quanto donato allo stato saturo, possiede un eccesso di intuizione che lo rende letteralmente incommensurabile. Al "soggetto" compete così di assumere la figura di colui che è investito e sommerso da questo eccesso di intuizione del fenomeno saturo: «ne consegue la nascita dell'adonato (l'adonné) soggetti(vi)tà interamente conforme alla donazione, che si riceve interamente da ciò che riceve» (MARION, 1997, p. 373). La figura dell'adonato, che - occorre ricordare - trova per Marion nella cogitatio cartesiana e nel rispetto kantiano due significativi antecedenti (cf. MARION, 1997, p. 375-389), inaugura pertanto una nuova conoscenza di sé da parte del "soggetto", la quale prende la forma del riconoscimento che l'ulteriorità della donazione si attesta come ciò che, nel momento in cui si dà (essendo irriducibile alle sue determinazioni ontiche), convoca il "soggetto" stesso a trascendere continuamente la specificazione ontica del suo stesso esserci. C'è un'indifferenza ad esserci per la quale l'uomo è chiamato a recuperare integralmente la figura autentica della sua umanità nell'appello che egli riceve a trascendere la caratterizzazione ontica della

3 Per quanto riguarda la traduzione da parte di Marion della Gegebenheit husserliana con donation col dibattito che ne è scaturito, cf. JANICAUD, D. La phénoménologie éclatée. Paris: L'éclat, 1998, p. 54-63; REALI. Fino all'abbandono, p. 51-52; 243-252; Idem. A proposito di “Dato che”. In: MARION, J. -L. Dato che. Saggio per una fenomenologia della donazione, tr. it., Torino: SEI, 2001, p. XV-XXIX. 
sua esistenza, quindi ad assumere quale tratto essenziale della sua realizzazione libera la dismisura del proprio compimento, che è tale in quanto imprevedibilmente manifestatagli da una donazione indeducibile da se stesso e dall'essere. Così facendo Marion ha l'ambizione di ripristinare l'affermazione centrale circa l'imprescindibilità di collocare la realizzazione libera dell'uomo solo all'interno della differenza aperta dall'ulteriorità della donazione, evitando di ricadere nelle aporie trascendentaliste ancora presenti nel pensiero heideggeriano dove il superamento dell'ontico è legato all'apertura strutturale all'Essere del Dasein inteso come l'ente nel quale ne va dell'Essere stesso (cf. MARION, 1991, p. 68-70; 91-106).

Così non deve suscitare meraviglia che Marion ritrovi nella donazione dei fenomeni "saturi" rivelati — eminentemente nel sacramento eucaristico - ciò che conduce la comprensione fenomenologica alla sua estrema possibilità. Dio, autorivelandosi, si rende presente nella storia attraverso fenomeni che sono "presso il soggetto" in una modalità che anticipa, non solo ogni pre-visione antropologica, ma anche il dono stesso, che all'uomo viene fatto, di poter prendere di mira l'autodonazione di Dio. Così la donazione non può mai affermarsi come una struttura formale entro la quale raccogliere, ancora una volta a priori, la comprensione del dono: il riconoscimento dell'uomo appartiene radicalmente a quanto di diritto eccede il campo della pre-visione, fosse anche quello del dono. $\mathrm{Si}$ tratta quindi di affermare che i fenomeni rivelati, nel pensiero di Marion, dicono sia il carattere di evento della rivelazione cristologica che compie «a priori l'a posteriori» (MARION, 1997, p. 388) della comprensione umana sia la sua discontinuità rispetto alle condizioni antropologiche in quanto tali. Tale discontinuità non sottolinea solamente che la comprensione permessa dall'autodonazione di Dio costituisce un'attuazione libera e, come tale, indeducibile dalle sue condizioni, ma anche - e più radicalmente - che l'avvenimento che la rende possibile è incommensurabile rispetto a queste condizioni. Il donarsi di Dio istituisce la verità della comprensione umana perché la pone come tale: il libero riconoscimento di colui che si consacra (s'adonne) all'evento cristologico non assicura il legame fra la condizione nella quale l'uomo è posto e il riconoscimento del suo senso, ma dice che il carattere di libertà del riconoscimento umano è già racchiuso e coinvolto nell'avvenimento della rivelazione stessa. 


\section{Dono-libertà}

La proposta è indubbiamente accattivante e può sicuramente fecondare il rapporto filosofia/teologia nella misura in cui tenta di evitare a teologi e filosofi l'affanno di una reciproca rincorsa che li fa giungere sfiatati ad un astratto punto di incontro. La marcata sottolineatura del principio della donazione ha, infatti, l'ambizione di assicurare uno spazio di dialogo tra le due discipline che, intrinseco al metodo fenomenologico stesso, permette di accostare i fenomeni rivelati proprio perché rivelati: questa loro identità garantisce, appunto, quello scarto (écart) tra il dato e il donato, tra l'apparenza e l'apparizione entro cui la donazione può affermarsi come irriducibile al soggetto e all'essere, e, pertanto, può configurarsi come ciò che di diritto appartiene al campo della fenomenalità, chiamando in causa direttamente il dinamismo libero dell'autodeterminazione umana. Ecco, allora, che quella che inizialmente sembrava essere un'ingenuità imperdonabile comincia a rivelare la sua fecondità dal momento che permette di approfondire il rapporto fenomenologia/teologia a partire da una problematica che focalizza immediatamente uno degli interessi principali sia della teologia contemporanea sia del filosofare stesso di Marion.

Nel rapporto dono-libertà, in gioco c'è infatti buona parte del recente rinnovamento metodologico della teologia, poiché l'abbandono della forma di pensiero moderna ha costretto il sapere teologico a volgere i suoi sforzi in direzione di una rivalutazione dell'esperienza contingente dell'uomo. Quest'ultima non può più essere considerata come una mera applicazione pratica dell'universale verità conosciuta per via concettuale, poiché - dall'incarnazione in poi - resta impossibile affermare la verità trascendente di Dio indipendentemente dal nesso che essa possiede con la tipicità del dinamismo storico dell'uomo. Semmai occorre mostrare continuamente, partendo dall'analisi delle modalità concrete dell'attuazione della fede, l'attinenza del dono della rivelazione cristologica con l'esperienza umana. In questo senso è chiaro, allora, che tematizzando il rapporto dono-libertà, si tratta, più radicalmente, di tentare di ri-pensare la relazione tra la 
trascendenza di Dio e la contingenza della libertà, in maniera tale che la differenza insuperabile che sussiste tra le due non diventi mai né una separazione né una coincidenza, ma, al contrario, istituisca un rapporto nel quale la verità trascendente di Dio possa porsi come effettiva garanzia della contingenza della libertà.

Per quanto riguarda Marion, il rapporto dono-libertà segnala un elemento fondamentale del suo percorso metodologico, giacché la forte sottolineatura del primato della donazione non deve far tuttavia concludere frettolosamente che egli s'indirizzi verso una sorta di monismo della donazione che accentua unilateralmente il momento manifestativo dei fenomeni. Il richiamo che Marion opera al dettato husserliano - in particolare al "Principio di tutti i principi" (MARION, 1997, p. 20-21) - mette bene in evidenza, infatti, come il darsi dei fenomeni sia inseparabile dalla loro attestazione nell'intuizione del soggetto, in modo tale che tutti i vissuti di coscienza appaiano come fenomeni a pieno diritto nella misura in cui sono dati ad una coscienza. Così, il fenomeno ridotto alla sua donazione opera un'effettiva liberazione dell'intuizione e dell'intenzione (cf. MARION, 1989, p. 48-57). Ciò che è dato alla coscienza e ciò che quest'ultima prende di mira è riunificato nella donazione, per cui non si tratta di opporre intuizione e intenzione, né di attribuire un primato all'una o all'altra, a partire dal quale recuperare successivamente un'unità persa in origine. È sufficiente conferire un primato assoluto e incondizionato alla donazione con cui guardare all'intuizione e all'intenzione come a due dimensioni della donazione stessa. Questo è possibile poiché ciò che fa accedere alla donazione è il gesto della riduzione, la quale consiste nel ricondurre "l'apparizione all'Io della coscienza e all'apparire stesso" (MARION, 1997, p. 27), che, detto diversamente, significa che i fenomeni si mostrano «come tali» quando il loro apparire è ricondotto dalla coscienza alla loro donazione la quale può così ricondurli fuori dall'Io per portarli a se stessi ${ }^{4}$. L'intuizione infatti, se primato deve avere, lo ha solo nel

\footnotetext{
4 "Se si ammette [...] che il fenomeno appare tanto più esso si dà perfettamente a vedere e a ricevere; ma anche che esso può darsi così solo se si dà all'lo della coscienza, dunque solo se si lascia ricondurre a quest'ultima - il che equivale a ridurvelo - allora resta da concludere che l'apparizione non si dona così perfettamente solo perché appare, quanto piuttosto perché si riduce alla sua donazione per la coscienza" (MARION, 1997, p. 26).
} 
senso che essa è donatrice del carattere donato del fenomeno, non perché la donazione sia misurata dall'intuizione, quanto perché quest'ultima è messa in opera dalla donazione affinché possa donarci la donazione stessa, la quale "al contrario dell'intuizione, [...] si riduce solo a se stessa e, quindi, si esercita assolutamente" (MARION, 1997, p. 28). Pertanto il reperimento del criterio idoneo a superare l'ideale moderno dell'oggettività, per Marion, si specifica adeguatamente solamente nel momento in cui si riconosce perfettamente legittimo evocare il primato della donazione - irriducibile al soggetto e all'essere - non separatamente dall'atto della soggettività umana che riconosce presente questa donazione nell'intuizione che essa ne ha: un'intuizione essa stessa data perché donata e donata in quanto data.

\section{Dono-gratuità}

Se così stanno le cose il rapporto dono-libertà rimane aperto su due versanti (quello teologico e quello fenomenologico) che da una parte e dall'altra sia ha l'ambizione di voler tenere uniti. Perché questo acca$\mathrm{da}$, occorre tuttavia mettere in atto un'operazione apparentemente paradossale: lasciare le due vie contraddittoriamente aperte, non soltanto nel senso che la teologia non può lasciare l'iniziativa alla filosofia (e viceversa), ma anche perché è palesemente impossibile scegliere chi tra le due deve dettare il cammino teoretico da intraprendere. Marion stesso ha cercato di percorrere questa strada. In parte lo si è or ora messo in evidenza: il dono si riduce alla sua donazione, e così ri(con)dotto dovrebbe avere la forza di tenere insieme il teologico e il filosofico senza nessuna ancillarità (né in un senso, né nell'altro). Tuttavia Marion sa bene che non è sufficiente affermarlo commentando il "principio di tutti i principi" husserliano, occorre individuare un fenomeno che abbia in sé tutte le caratteristiche di un dono di questo tipo, perché altrimenti si corre un rischio che comprometterebbe l'intero progetto di una fenomenologia della donazione: «la pretesa riduzione fenomenologica del dono alla donazione alla fine non gli interdice [al dono stesso nda] la dignità stessa di fenomeno?» (MARION, 2010, p. 161). In altri termini, il dono ridotto alla 
donazione ha sì cercato di strappare gli ormeggi del concettualismo e del trascendentalismo moderno, ma deve pur sempre conservare con scrupolo e attenzione un legame con il mondo dell'effettività per non scomparire come «un noumeno refrattario alle condizioni dell'esperienza» (MARION, 2010, p. 143). Ebbene, un fenomeno, meglio un dono, di questo tipo per Marion esiste e prende un nome preciso: la paternità.

Il percorso della fenomenologia di Marion determina in questo modo un ulteriore fattore di interesse, che rende sempre più evidente la plausibilità di un suo approfondimento da parte della teologia. Approfondimento da condursi, tuttavia, avendo la preoccupazione di non trarre frettolosamente deduzioni apologetiche; come accadrebbe, per esempio, se si volesse subito intravedere il punto di arrivo del percorso di Marion, accentuando l'idea — per altro incontestabile - che la sessualità umana è realmente tale solo quando assume la forma della paternità. Un'ingenuità di questo tipo nessun teologo serio la compirebbe, sapendo bene che, comunque, resta il problema (meglio il dovere) di mostrare le ragioni (in senso biblico, naturalmente, cf. 1Pt 3,15) di simile affermazione. Alla stesso modo la teologia deve anche fare attenzione a non trarre un'altra sbrigativa conclusione: scorgere nel suggerimento di Marion la via attraverso cui "risolvere" il problema della sessualità mediante il ricorso ad una concettualità di tipo univoco che finisce per rendere sinonimiche le categorie di paternità e gratuità. Questo accade quando si tenta di "spiegare" la prima ingenuità segnalata, producendo un'argomentazione grossolanamente sintetizzabile in questo modo: la sessualità è se stessa solo nella forma della paternità, e la ragione di simile affermazione sta nel fatto che la paternità si configura come un dono gratuito. Qui il ragionamento - perché di ragionamento si tratta - , seppur mosso dal desiderio di non fermarsi alla dichiarazioni apodittiche, sarebbe ancor meno efficace che nel primo caso, poiché non solo sottometterebbe l'esperienza umana alle forche caudine di una deduzione concettuale, ma anche perché si discosterebbe alquanto dal pensiero di Marion. Quest'ultimo - rispondendo alle critiche di Derrida, secondo cui il dono resta sempre prigioniero dell'economia dello scambio (cf. DERRIDA, 1991) ${ }^{5}$ - è, infatti, dell'opinione che implicare la

5 In proposito cf. On the Gift. A Discussion between Jacques Derrida and Jean-Luc Marion. In: CAPUTO J. D.; SCANLON, M. J. God, the Gift and Postmodernism. Bloomington/Indianapolis: Indiana University Press, 1999, p. 54-78. 
gratuità nel dinamismo del dono porta con sé il grave pericolo di privare il dono stesso della dimensione dell'intersoggettività, quindi della condizione essenziale al suo accadere fenomenico. Ciò avviene nella misura in cui la gratuità, specialmente laddove è più perfetta ed assoluta possibile, esige non solo che scompaia ogni forma di contraccambio, ma che svanisca persino l'altro al quale si dona: si dovrebbe donare senza sapere a chi si dona, anzi si dovrebbe donare senza sapere che non si dona a nessuno, e solo così il dono si sottrarrebbe all'economia. Ma in questo caso, «come per un giansenismo e un pietismo fenomenologico, si interdice al dono l'orizzonte stesso del suo dispiegamento fenomenale - l'alterità stessa» (MARION, 2010, p. 162): la pretesa purezza del dono svela un'indipendenza dall'altro-da-sé equivalente ad un'autarchia altrettanto assoluta che, anziché garantire l'autenticità del dono, ne interdirebbe l'effettuazione. Per di più Marion è convinto che la gratuità non appare affatto come risolutiva dell'identità del dono, perché essa, seppur rivendicata come il miglior rimedio contro l'economia dello scambio, di fatto finisce sempre per essere il tentativo di trovare una ragione e/o una causa al dono, e, proprio per questo, così come l'economia, appartiene alla razionalità metafisica moderna: in entrambi i casi «si tratta di rendere ragione e di pensare rendendo ragione» (MARION, 2010, p. 162). Dal suo punto di vista la gratuità come causa del dono rappresenta il sostenitore cosciente e ben determinato del mondo dell'economia, che pure cerca di fare in modo che venga radicalmente interrotto e dimenticato, e questo perché - vale la pena ripeterlo nuovamente - la gratuità si configura come il motivo o la causa che, tentando di rendere ragione del dono, si sottomette alla ragione sufficiente dell'economia: «l'economia fa l'economia del dono, poiché essa ne rende sufficientemente ragione» (MARION, 2010, p. 154).

Così facendo, Marion, ancora una volta, si preoccupa di identificare nella lotta al razionalismo metafisico moderno l'elemento costitutivo (in negativo) del suo discorso. "Spiegare" la donazione del dono significa imporre a priori dei paletti al campo della fenomenalità, il che corrisponde al percorso che la modernità, da Descartes fino ad Hegel, ha compiuto radicalizzando il principio di ragion sufficiente: «tutto ciò che è, è in quanto una causa (effettività) sive ratio (concetto) rende ragione sia della sua esistenza, sia della sua inesistenza, sia della sua indipendenza da 
qualsiasi causa» (MARION, 1989, p. 426; 1992, p. 80-83; 1997, p. 253255). Tale principio, imponendo pertanto di assegnare ad ogni fenomeno il suo concetto o la sua causa (causa sive ratio), finisce per togliere dignità fenomenica a tutti i fenomeni irriducibili ad una causa concettualizzante. Di conseguenza la difficoltà maggiore con la quale la riflessione sul dono deve misurarsi non sta tanto nell'invocare un "dono senza economia" appellandosi all'idea di gratuità - quanto nel riconoscere che esiste un "dono senza spiegazione". In altri termini, per Marion, occorre mostrare la legittimità di un percorso fenomenologico che riconosce come effettivamente donati tutti i fenomeni dati, ossia tutti i fenomeni che non hanno bisogno di un motivo o una causa per diventare intelligibili (soddisfacendo così la ragione sufficiente), ma che dati nell'effettività del campo della fenomenalità sono ad esso donati: ridurre il dono alla donazione.

\section{Dono-paternità}

È un problema, quindi, di identificazione di un percorso metodologico interno alla fenomenologia, quello che assorbe lo sforzo teoretico di Marion, il quale si ritrova così allo stesso punto dal quale era partito. Il problema iniziale di sottrarre la sua prospettiva di pensiero all'obiezione dell'irrilevanza del carattere fenomenico di un dono così concepito, lo conduce ad evocare la necessità di illustrare e chiarire che il dono ridotto alla donazione non è un "noumeno refrattario all'esperienza". Ciò, nel momento in cui incrocia le obiezioni di Deridda circa l'impossibilità di uscire dall'orizzonte dell'economia, lo porta a rinnovare e riproporre l'idea che l'esclusione radicale della prospettiva economica sia possibile solo laddove si riaffermi il criterio metodologico elaborato: al contrario della gratuità, che è, anzitutto, l'offerta di un razionalismo camuffato, per Marion, ridurre il dono alla donazione è lo strumento criteriologico che ha in sé la forza necessaria ad affrancare il dono dall'orizzonte dell'economia. Ma, come per un'inesplicabile destino, il cammino percorso sente il bisogno di aprirsi di nuovo all'effettività fenomenica identificando nella paternità l'exemplum significativo di che cosa di fatto significhi un dono ridotto alla donazione: «un dono da sempre già ridotto alla donazione e 
franco da ogni scambio nell'economia. Che fenomeno soddisfa queste esigenze? Noi ne suggeriamo uno - la paternità» (MARION, 2010, p. 162). Quest'ultima è il fenomeno che Marion mette in gioco con l'intento di indicare che, essendo condizionato pesantemente ovvero addirittura chiuso ogni altro spazio di legittimazione fenomenica del suo discorso e di sviluppo tematico, non rimane che volgersi all'effettività, che è luogo ancora libero e frequentabile per far apparire la plausibilità del suo pensiero. La paternità costituisce, così, l'ambito dove concentrare lo sforzo di ristabilire il pensiero pensato nella storicità effettiva dei fenomeni, stando attenti, tuttavia, a mantenere costante lo sguardo sul pensiero per ritrovare nel fenomeno della paternità tutte le caratteristiche di ciò che il criterio metodologico ha identificato in un dono ridotto alla donazione.

Così facendo, per un supremo paradosso, l'effettività offre di nuovo una possibilità al pensiero, ma subito deve arretrare per lasciare il passo al ragionamento condotto. Prova ne è che Marion, introducendo una puntuale disamina del fenomeno della paternità, si preoccupa immediatamente di affermare che essa ha l'identità di un avvenimento che «non fabbrica un risultato finito, determinato e terminato una volta consegnato, ma provoca un possibile (il figlio) il cui avvenire non si può a sua volta né prevedersi, né dedursi dalle sue cause, né anticiparsi, ma deve attendersi» (MARION, 2010, p. 163-164). La paternità, cioè, è sì un avvenimento che accade, ma il suo accadere non permette l'accesso all'avvenimento stesso, poiché la sua fenomenalizzazione (l'aver generato) non basta a rendere conto del futuro possibile che deve ancora accadere. Esiste, allora una differenza tra il padre e la paternità, che ricalca letteralmente lo scarto (écart) teoreticamente asserito tra il dato e il donato; e, come in quest'ultimo caso, la differenza era stabilita per far fuori definitivamente ogni prospettiva che cercasse di com-prendere i fenomeni a partire da qualcos'altro rispetto alla loro donazione (per cui si doveva stabilire che ogni dato è donato), anche per quel che concerne la paternità, si deve decretare che si tratta di un fenomeno dato in quanto donato, ossia che riconosce solo nella donazione l'unica autorità legittimata a "comprendere" la sua apparizione fenomenale (diversamente indicibile e in-comprensibile). Ciò nonostante Marion sa bene - come abbiamo già sottolineato - che il donato deve anche darsi (pena il suo dissolvimento fenomenico come un 
"noumeno refrattario all'esperienza"), per questo, allora, il punto di riferimento col quale leggere l'avvenimento della paternità deve essere continuamente spostato al fatto che «il fenomeno donato qui dà [...] il donato non solamente si dà, ma dà un altro donato diverso da sé» (MARION, 2010, p. 164). Quest'ultimo è senza dubbio il figlio che, generato dal padre (e a sua volta possibile padre), innesca il meccanismo della donazione dentro il flusso della storicità, in modo tale che la precisazione di Marion sembrerebbe interrompere la stesura del ragionamento per fornire al disegno teoretico una qualche prospettiva storica, un'animazione e un movimento di piani e tempi più complessi del semplice criterio epistemologico della donazione. Tuttavia ciò che colpisce, qui, è ancora una volta la forza del pensiero che si avverte nella scrittura di Marion di fronte alla storicità: la ricerca della novità a cui apre la generazione ha bisogno di aiuti, di soccorsi, di appoggi, tanto il carattere fattuale della paternità non gli sembra, nella sostanza, di per sé autorevole e significativo per specificare cosa significhi un dono ridotto alla donazione. Marion deve, quindi, associare al flusso della storia aperta dal fenomeno della paternità, la sottolineatura che la novità generata (il figlio) ha l'identità — ancora una volta - del donato, il che equivale a dire che la possibilità del donato di darsi (e quindi di fenomenalizzarsi) è innescata dalla donazione stessa, in modo tale che il donato si dà esclusivamente per ri-dare la donazione.

Una volta chiarita la struttura del dono ridotto alla donazione, l'esortazione fenomenologica di Marion diventa particolarmente perentoria nell'ulteriore approfondimento del fenomeno della paternità che viene a completare il cammino fin ora percorso. Il passo è compiuto a partire dalla consapevolezza che la donazione, essendo irriducibile alla sua datità, non per questo produce uno stile filosofico che assorbe il dato nel donato. Tale nuova possibilità è innescata dalla donazione stessa nella misura in cui «non c'è nulla che si mostri se, innanzi tutto, non si è donato» (MARION, 2001, p. 148), ossia se nella fenomenalizzazione del fenomeno - che avviene solo a partire da sé e tramite sé - «ciò che appare si dà nel suo apparire» (MARION, 2001, p. 148). La res del fenomeno si dà dunque interamente nella sua donazione, e, dandosi totalmente in essa, dona contemporaneamente al dato la possibilità di darsi, con la significativa avvertenza che tutto ciò accade a posteriori della donazione. La 
possibilità è quindi concessa sulla base del ri-conoscimento che solo nell'a priori della donazione è incluso l'a posteriori del darsi del dato, in modo tale che quest'ultimo, essendo sempre in posizione di ricevere - debba ricevere anche se stesso da ciò che riceve: «questo fenomeno [la paternità nda], che si dà donando, può a sua volta darsi solo se si scopre anzitutto donato a se stesso» (MARION, 2010, p. 164).

L'essere anzitutto figli per poter essere padri, è così il livello nel quale il pensiero di Marion rivela la volontà di andare fino in fondo nel cammino intrapreso, e lo fa rivelando come la donazione costituisca la matrice a cui guardare per illuminare il dato. Ciò significa che Marion ha ferma la convinzione che il fenomeno della paternità (donato) non possa essere pienamente riconosciuto nella sua effettiva identità quando l'essere padre (il darsi del dato) dimentica di essere a sua volta originato e permesso dalla donazione (donato a se stesso). In questo modo il dato è ancor più ri(con) dotto alla donazione, tuttavia l'indicazione resterebbe su un piano troppo formale se non si spingesse a mettere in evidenza la qualità specifica e concreta che al dato (in questo caso al padre) è richiesta per essere colui che «si riceve egli stesso da ciò che riceve» (MARION, 1999, p. 20). Ecco, allora, che l'affermazione secondo cui "non c'è nulla che si mostri se, innanzi tutto, non si è donato" prende la forma di un avvenimento in cui il «padre per dimorare deve mancare, dunque brillare per la sua assenza» (MARION, 2010, p. 165). La presenza del padre alla vita del figlio, per Marion, diventa ancor più incisiva laddove scompare (o per lo meno si riduce il più possibile) la componente fisico-materiale del suo essere padre. L'impossibilità di delimitare il significato della paternità alla sfera puramente ontico-materiale della presenza del padre è così legata alla volontà di sottolineare come la paternità stessa è irriducibile alla sua determinazione ontica (il dato di essere padre), per potersi di conseguenza aprire verso una caratterizzazione che integra quell'aspetto di ulteriorità a partire dal quale può essere decretato il primato della donazione. Non a caso Marion esemplifica il suo pensiero soffermandosi sull'istante dell'atto della procreazione dove, ai suoi occhi, appare più evidente l'insuperabile scarto che esiste tra il dato e il donato: «Il padre manca. Egli manca anzitutto perché procrea solo nell'istante e, divenuto inutile, subito si ritira diversamente dalla madre che resta e nella quale il figlio rimane» (MARION, 2010, p. 165). La paternità nasce e si 
sviluppa contemporaneamente all'allontanarsi del padre, al rendersi esile, incerto (terminando) il darsi del dato, così che quest'ultimo manifesti una differenza dal donato che, nella sua insuperabilità, lungi dal proporsi come ostacolo al crescere della paternità nei confronti del figlio, diventa garanzia della sua effettuazione ${ }^{6}$. Allo stesso modo il figlio deve sperimentare anch'egli questo inesorabile scarto tra il dato e il donato, assumendo l'impossibilità a restituire ciò che ha ricevuto dal padre (donato) come la "condizione" essenziale del suo essere figlio (dato):

Non si tratta di ingratitudine soggettiva [...] Si tratta, più radicalmente, di un'impossibilità di principio: che lo voglia o no, che vi si senta obbligato o meno, il figlio non potrà mai "restituire" e resterà ingrato, insufficiente e incosciente, perché egli non potrà mai rendere a suo padre ciò che ha da lui ricevuto - la vita (MARION, 2010, p. 166).

La vita sostituisce, così, ogni dominio delle discipline sperimentabili sulla paternità, poiché quest'ultime non colgono il vivere se non dissecandolo e uccidendolo, e la verità dell'essere padre (nei sentimenti, nelle passioni, nei contrasti, in breve nella sua dimensione spirituale) non può che apparire morta nella fredda analisi della sperimentazione, infinitamente limitata al solo esame del dato, là dove, invece, la vita la mantiene aperta al sovrappiù inoggettivabile della paternità donata:

il dono dato qui si riduce precisamente alla vita, che [...] non dipende né dall'entità, né dall'oggettività. La vita non è poiché niente è senza essa. Nessuno la vede, né la definisce, né l'afferra come qualcosa di reale [...] Il cadavere d'altronde non manca di nulla di reale che permetterebbe di distinguerlo dal vivo (MARION, 2010, p. 168).

La paternità nel pensiero di Marion vuole, insomma, essere tale consacrandosi alla vita che la libera da ogni compromissione con l'ontico e con le scienze esatte (che dominano questo regno) e la fa durare per sempre nella condizione di dono dato. La paternità vuole, in altre parole, fissarsi nella perfezione assoluta della vita, quindi della donazione. Proprio

6 «Solo la distanza, mantenendo uno scarto distinto tra i termini [...] rende possibile la comunione, e media immediatamente la relazione" (MARION, 1991, p. 239). 
per questo Marion propone fortemente lo sradicamento dall'ontico come paradigmatico della paternità e si sforza di contrapporlo al permanere della madre accanto al figlio: è consapevole dell'irrilevanza della particolarità della scelta del padre, ma ritiene necessario rendere nota al lettore la conclamata esigenza che tale scelta si dia nella particolarità.

Il problema per Marion è quello di una filosofia che possa presentare la varietà e l'infinita potenzialità della vita senza distruggerla nella forma: ben consapevole che una filosofia del genere non deve moltiplicare i punti di vista, ma piuttosto annullarli completamente nella definitiva assenza di punti vista (cioè della singola coscienza del dono). Marion compie, in questa prospettiva, il cammino inverso rispetto all'esperienza consueta e più diffusa della paternità. Al contrario di quest'ultima, il padre di Marion dissolve se stesso e cancella la stessa esperienza dell'essere padre che, in un paradosso voluto, quando comincia a raccontare, già possiede. La fenomenologia della donazione di Marion è il racconto di tale spogliazione, ma non secondo una trama di rapporti e circostanze che accadono nell'esperienza, bensì in una sorta di dimissione annunciata: non la vita, quindi, ma la perfezione della donazione, riperfezionata ogni volta dal fatto di crederci fino in fondo e di investirsene totalmente, con pieno abbandono, come se fosse vita, senza, però, che vita sia, cioè sempre col distacco del filosofo che sa di poter far accadere ciò che non è mai accaduto e che, dopo, può scoprire come non avvenuto, di far apparire ciò che la donazione dà, ma senza che nulla si modifichi davvero, e allora ancora infinite altre volte l'esistenza può essere moltiplicata nella donazione in modi sempre nuovi, dal momento che in ogni momento la donazione può rinnovare, trasformare i particolari e le situazioni della vita, senza che nulla di ciò che è venga turbato, poiché in fin dei conti, per Marion, nella donazione "niente è, ma tutto appare".

\section{Riferimenti}

DERRIDA J. Donner le temps. 1. La fausse monnaie. Paris: Galilée, 1991.

JANICAUD, D. La phénoménologie éclatée. Paris: L'éclat, 1998. 
MARION, J. L. Réduction et donation. Recherches sur Husserl, Heidegger et la phénoménologie. Paris: Presses Universitaires de France, 1989.

MARION, J. L. Étant donné. Essai d'une phénoménologie de la donation. Paris: Presses Universitaires de France, 1997.

MARION J. L. Dieu sans l'être. Paris: PUF, 1991.

MARION, J. L. Dato che. Saggio per una fenomenologia della donazione. Trad. it., Torino: SEI, 2001.

MARION, J. L. Le phénomène saturé. In: HENRY, M., et al. Phénoménologie et théologie. Paris: Criterion, 1992.

MARION, J. L. Filosofia e Rivelazione. Revista Studia Patavina, Padova, v. 36, p. 426, 1989.

MARION, J. L. L'événement, le phénomène et le révélé. Revista Transversalité, Paris, v. 70, p. 20, 1999.

MARION, J. L. La fenomenalità del sacramento. Essere e donazion. In: REALI, N. Il mondo del sacramento. Teologia e filosofia a confronto. Milano: Paoline, 2001.

MARION, J. L. L'inconditionné ou la force du don. In: MARION, J. L. Certitudes négatives. Paris: Grasset, 2010.

REALI, N. Fino all'abbandono. L'eucaristia nella fenomenologia di Jean-Luc Marion. Roma: Città Nuova, 2001.

Ricevuto: 29/06/2016

Received: 06/29/2016

Approvato: $15 / 08 / 2016$

Approved: 08/15/2016 